in subcortical white matter of frontal lobes and basal ganglia, without mass effect. Disseminated toxoplasmosis was diagnosed at autopsy, and microscopy showed necrotizing toxoplasmic encephalitis. (Tsuge I, Matsuoka H, Nakagawa A et al. Necrotizing toxoplasmic encephalitis in a child with the X-linked hyper-IgM syndrome. Eur I Pediatr Sept 1998;157:735-737). (Respond: Dr I Tsuge, Department of Paediatrics, Nagoya University School of Medicine, Tsurumai-cho 65, Showa-ku, Nagoya 466, Japan).

COMMENT. X-linked hyper-IgM syndrome is an immunodeficiency disorder characterized by recurrent infections with opportunistic organisms, such as Pneumocystis carinii and Cryptosporidium, and associated with low serum levels of $\operatorname{IgG}$ and $\operatorname{IgA}$, and normal to increased $\operatorname{IgM}$. Disseminated toxoplasmosis is now added to the potential complications of this disorder.

\title{
CEREBRAL TUMORS
}

\section{INTRACRANIAL ARACHNOID CYSTS AND ASSOCIATED LESIONS}

The clinical manifestations and associated brain anomalies of intracranial arachnoid cysts are reported in 30 children treated at the National Taiwan University Hospital, Taipei. Onset of symptoms ranged from 1 day to 14 years (mean, 4 years, 7 months), and age at diagnosis was 10 days to 16 years (mean, 6 years, 2 months). The majority of cysts were located in the middle fossa, usually unilateral (17 cases); 7 were in the posterior fossa. Presenting clinical symptoms included seizures in 11 cases (37\%), headache in 6 (20\%), and macrocephaly (2 cases). EEG showed focal temporal or central epileptiform discharges in 13 of 17 patients. In patients with intractable seizures, surgery resulted in only partial reduction in cyst size and seizure frequency. Progressive hydrocephalus and increased intracranial pressure occurred in 2 patients and were relieved by ventriculo-peritoneal shunt. Associated anomalies included brain tumor, nevocellular nevi, achondroplasia, microphthalmia, intracystic hemorrhage, corpus callosum dysgenesis, and heterotopia. (Wang P-J, Lin H-C, Liu H-M, Tseng C-L, Shen Y-Z. Intracranial arachnoid cysts in children: related signs and associated anomalies. Pediatr Neurol Aug 1998;19:100-104). (Respond: Dr Wang, Department of Pediatrics, National Taiwan University Hospital, 7 Chung-Shan South Road, Taipei, Taiwan).

COMMENT. Intracranial arachnoid cysts (AC) may be asymptomatic and are recognized incidentally by CT or MRI for head trauma or at autopsy. Some are identified by neuroimaging performed because of headache, seizures, ataxia, or macrocephaly. The pathophysiology is a congenital malformation of the leptomeninges. The temporal lobe or middle cranial fossa is the most common location. The conservative approach to treatment, usually favored by neurosurgeons, is based on lack of sustained benefit and a 30\% risk of cyst recurrence after surgery.

In addition to headaches and seizures, the most common presenting symptoms of AC, attention deficit hyperactivity disorder has been associated with temporal lobe AC, the TAC/ADD syndrome. (Millichap JG. Temporal lobe arachnoid cyst - attention deficit disorder syndrome: role of the electroencephalogram in diagnosis. Neurology May 1997;48:1435-1439). A causal association was considered plausible based on the coincidental learning and language disabilities that might be explained by temporal lobe and sylvian region pathology in the the initial 3 cases reported. Since this publication, I have diagnosed one further case and colleagues have provided me with case reports of 3 examples of the TAC/ADD 
syndrome. The EEG was normal in 2 and at follow-up in one other whose initial record had shown focal temporal slow and sharp waves. Cases of AC involving the temporal lobe warrant psychological testing for associated ADHD and learning disorders.

\section{CEREBELLAR VERMIS SPLIT SYNDROME}

Surgical transection of the posterior inferior cerebellar vermis in 5 children (ages 6 - 15 years) treated at Washington University School of Medicine, St Louis, MO, was followed by selective impairment of tandem gait. Surgery performed for removal of tumors in the fourth ventricle involved destruction of the midline parallel fibers ranging from lobules VI-X. Regular self-paced gait, Romberg posture, and hopping were only minimally impaired, and kicking, reaching, or speech were unaffected. (Bastian AJ, Mink JW, Kaufman BA, Thach WT. Posterior vermal split syndrome. Ann Neurol Oct 1998;44:601-610). (Respond: Dr Bastian. Program in Physical Therapy, Washington University School of Medicine, 4444 Forest Park Parkway, Box 8502, St Louis, MO 63108).

COMMENT. Profound and persistent ataxia of tandem gait occurs after surgical division of midline fibers crossing the posterior inferior vermis of the cerebellum, whereas regular gait, hopping, individual limb coordination, and speech are relatively unaffected. Damage to both vestibular and visual inputs subserved by the posterior midline vermis fibers may explain the preferential impairment of tandem gait.

Vermian agenesis without posterior fossa cyst or fourth ventricle enlargement was diagnosed by MRI at 16 months to 5 years in 14 children presenting with oculo-motor apraxia, ataxia, and global developmental delay at Saint-Vincent de Paul Hospital, Paris, France (Nabbout R, Bulteau C et al. Brain Dev Sept 1998;20:429), and presented at the VIII International Child Neurology Congress, Ljubljana, Slovenija, Sept 1998.

\section{SEIZURE DISORDERS}

\section{EFFICACY OF KETOGENIC DIET AGAIN RE-VISITED}

A prospective multicenter study of the efficacy of the ketogenic diet in treatment of 51 children with intractable seizures is reported from 6 large institutions, including Johns Hopkins, Montefiore and Boston, USA, and Halifax, Canada, and 1 private practice setting. A 4:1 ketogenic diet was introduced in hospitalized patients after 36 hours fasting, according to the Johns Hopkins protocol. Pre-diet parental estimates of seizure frequencies were compared with seizure occurrences after 3, 6, and 12 months treatment. A 50\% decrease in seizure frequency or greater was observed in $54 \%$ at 3 months, in $55 \%$ at 6 months, and in $40 \%$ at 1 year. Only 5 patients were seizure-free at 1 year. The fall out rate of patients starting the diet was $12 \%$ at 3 months, $31 \%$ at 6 months, and $53 \%$ at 1 year. Reasons for discontinuance of the diet were medical intolerance due to lethargy, dehydration, vomiting, or behavioral problems in 6 , the restrictive dietary protocol in 4, and lack of seizure control in 12. Seizure type (tonic-clonic in 20, myoclonic (19), atonic (14), absence (8), and partial (9)) and EEG abnormality (generalized or multifocal epileptiform) were not related to outcome. (Vining EPG, Freeman JM, Ballaban-Gil K, et al, and the Ketogenic Diet Multi-Center Study Group. A multicenter study of the efficacy of the ketogenic diet. Arch Neurol Nov 1998;55:1433-1437). (Respond: Eileen PG Vining MD, The Johns Hopkins Medical 